\title{
SURGICAL EXCISION OF MYCOTIC (CLADOSPORIUM SP.) GRANULOMAS FROM THE MANTLE OF A CUTTLEFISH (SEPIA OFFICINALIS)
}

\author{
Craig A. Harms, D.V.M., Ph.D., Dipl. A.C.Z.M., Gregory A. Lewbart, M.S., V.M.D., Dipl. \\ A.C.Z.M., Ryan McAlarney, Larry S. Christian, Kyleigh Geissler, D.V.M., and Carol Lemons
}

\begin{abstract}
An adult female European cuttlefish (Sepia officinalis) from a public aquarium presented with an eruptive skin lesion of the dorsal mantle. Radiographs, hemolymph collection, and excisional biopsy were performed using anesthesia with ethanol $1.5-3 \%$ in seawater. Elastic and freely mobile skin permitted closure with minimal tension following wide excision around the lesions, which did not appear to penetrate deep to the underlying cuttlebone. Biopsy revealed hemocyte granulomas surrounding thin, septate, infrequently branching fungal hyphae, and culture yielded Cladosporium sp. Itraconazole was administered postoperatively in food items. The cuttlefish recovered to normal feeding and activity levels with complete surgical site healing. Two months following the procedure, the animal was found dead in exhibit. Histopathologic examination revealed multisystemic fungal infection.
\end{abstract}

Key words: Cladosporium, cuttlefish, Sepia officinalis, surgery.

\section{INTRODUCTION}

Cuttlefish are popular animals in public and private aquarium displays and have been used as laboratory and food animals. Like other cephalopod mollusks, such as octopus and squid, cuttlefish are characterized by a highly developed neurological system and complex behaviors, contrasted with a relatively brief lifespan and death typically following shortly after reproduction. ${ }^{1}$ Disease, husbandry, and therapeutics of cephalopods have received attention in recent years. $3,4,6,11-13,15,17,18,20$ The internal shell of cuttlefish, the "cuttlebone," can sustain fractures and infections which can prove fatal. ${ }^{17,18}$ Fungal diseases of cephalopods are not commonly reported, but include Fusarium sp. in chambered nautilus (Nautilus pompilius) and Cladosporium sp. in an unspecified octopus species. ${ }^{17}$ This report describes anesthesia and surgical treatment of a Eu-

From the North Carolina State University, College of Veterinary Medicine, Department of Clinical Sciences, Center for Marine Sciences and Technology, 303 College Circle, Morehead City, North Carolina 28557, USA (Harms); North Carolina State University, College of Veterinary Medicine, Department of Clinical Sciences, 4700 Hillsborough St., Raleigh, North Carolina 27606, USA (Lewbart, Christian); North Carolina Aquarium at Fort Fisher, 900 Loggerhead Road, Kure Beach, North Carolina 28449, USA (McAlarney); North Carolina State University, College of Veterinary Medicine, Department of Population Health and Pathobiology, 4700 Hillsborough St., Raleigh, North Carolina 27606, USA (Geissler); and North Carolina State University, Clinical Microbiology, Veterinary Teaching Hospital, 4700 Hillsborough St., Raleigh, North Carolina 27606, USA (Lemons). Correspondence should be directed to Dr. Harms. ropean cuttlefish (Sepia officinalis) with mycotic dermatitis of the dorsal mantle caused by Cladosporium sp. infection.

\section{CASE REPORT}

An adult female captive cuttlefish (220 g) with a history of an acute eruptive skin lesion of the dorsal mantle was presented for examination. The lesion began as a thickening and darkening of the skin, and progressed to partial ulceration. The cuttlefish had been obtained 5 mo previously at an estimated age of 4-6 mo along with 11 others, 8 of which remained and appeared healthy. Cuttlefish were kept in a 3,400-L artificial seawater tank (waterquality ranges throughout holding: $\mathrm{pH} 7.8-8.1$, salinity $33-35 \mathrm{psu}$ (ppt), ammonia $0.01-0.18 \mathrm{mg} / \mathrm{L}$, nitrite $0.07-0.12 \mathrm{mg} / \mathrm{L}$, nitrate $0-176 \mathrm{mg} / \mathrm{L}$, temperature $21-23^{\circ} \mathrm{C}$ ). Feeding and activity levels were unchanged from prior to development of the skin lesions. The week prior to presentation, a swab of the ulcer for culture and sensitivity and a cytology smear had been submitted to an outside laboratory (Antech Diagnostics, Southaven, Mississippi 38671, USA). Culture yielded moderate growth of an alpha hemolytic Streptococcus sp. sensitive to all antibiotics tested. Cytology showed a highly cellular hemocytic response surrounding fungal hyphae that were grey, septate, and branching, with blunt ends, and measuring approximately $4 \mu \mathrm{m}$ wide. Prior to receiving cytology results, treatment with enrofloxacin (Baytril, $22.7 \mathrm{mg} / \mathrm{ml}$, Bayer Corporation, Animal Health, Shawnee Mission, Kansas 66216, USA) $10 \mathrm{mg} / \mathrm{kg}$ p.o. q $12 \mathrm{~h}$ in food items ${ }^{4}$ was attempted, but resulted in feed refusal.

At the time of presentation, the original lesion 


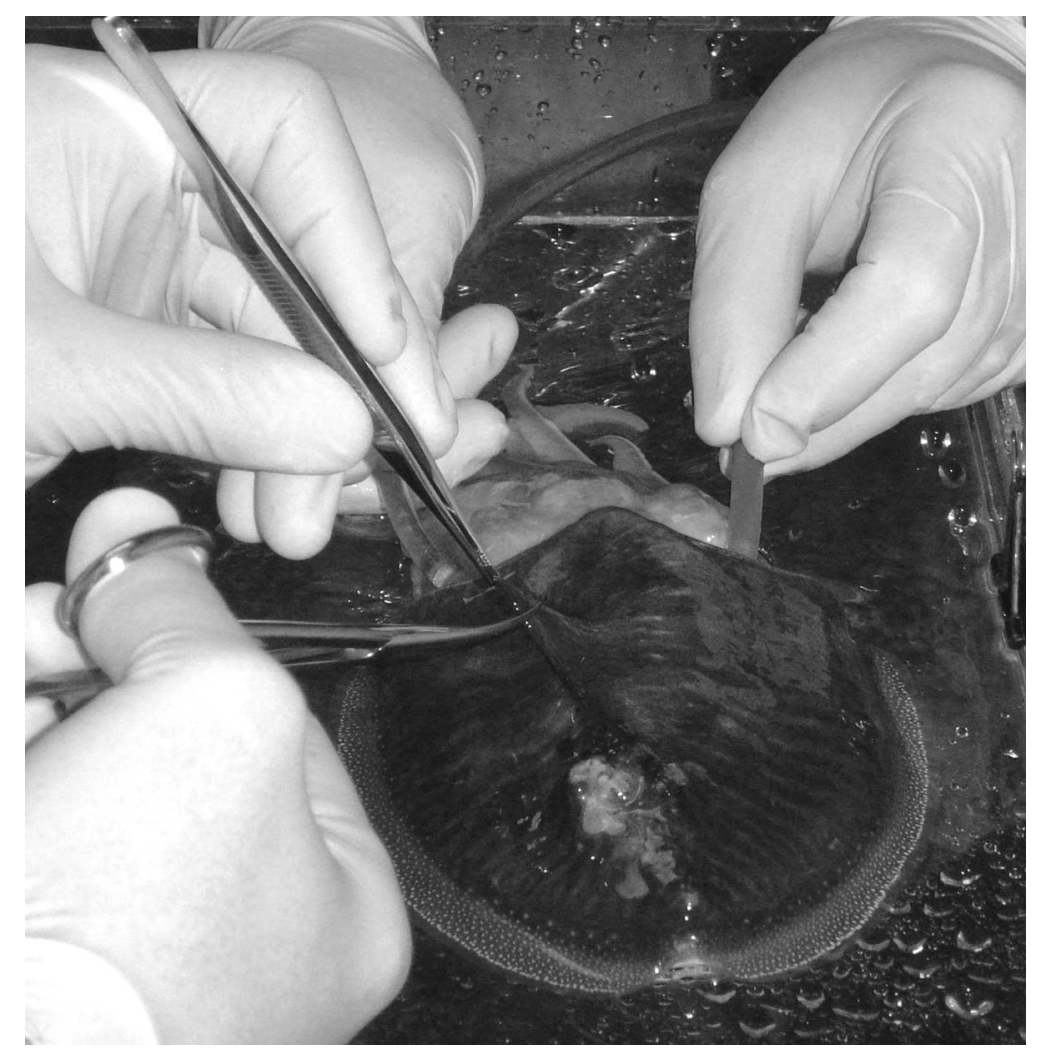

Figure 1. Cuttlefish on recirculating anesthesia machine during surgery, showing double-tube delivery of ethanolcontaining seawater bilaterally to both ctenidia (gills), and mobility of skin for simple excision of the smaller skin nodule.

had an open ulcer approximately $1.5 \mathrm{~cm}$ diameter with underlying muscle exposed and a necrotic core, but no cuttlebone exposure. It was located dorsally on the left side of the mantle near the apex. Craniomedial to and confluent with the original lesion was an irregular dark firm thickened area. The combined lesions measured approximately $2.5 \times$ $3.0 \mathrm{~cm}$. One isolated firm subcutaneous nodule was present further cranially, just left of midline near the middle of the mantle, measuring approximately $4 \mathrm{~mm}$ diameter. All lesions were freely mobile and not attached to the underlying mantle musculature.

The cuttlefish was anesthetized for radiographs, hemolymph collection, and excisional biopsy, using ethanol $30 \mathrm{ml} / \mathrm{L}(3 \%)$ in seawater. ${ }^{14}$ Induction was rapid, within $1 \mathrm{~min}$, at which point the ethanol concentration was reduced to $15 \mathrm{ml} / \mathrm{L}(1.5 \%)$ by adding anesthesia-free seawater. A dorsoventral radiograph was obtained by removing the cuttlefish from the anesthesia water, placing it directly on a film cassette for image acquisition (52 kvp, $1.0 \mathrm{mAs}$, Kodak X-Omatic cassette with Kodak Lanex medium screens and Kodak TMat G film, Rochester,
New York 14652, USA), and returning it to the anesthesia water within $1 \mathrm{~min}$. For maintenance and the remaining diagnostics, the cuttlefish was transferred to a recirculating anesthesia system on a smooth nonabrasive acrylic surgical platform ${ }^{9}$ with two independent tubes delivering anesthesia water bilaterally into the mantle cavity over both ctenidia (gills) (Fig. 1). Respirations continued sporadically throughout the procedure, but the cuttlefish remained unresponsive to tactile and surgical stimuli. Hemolymph $(0.5 \mathrm{ml})$ was collected from the muscular portion of the cephalic vein just dorsal to the funnel with the use of a 1-ml syringe with 25-ga 9-mm needle for hemocyte count (manual count with hemacytometer and no staining), hemocyte cytology (Wright-Giemsa stained hemolymph smear) and hemolymph chemistry panel (Roche/Hitachi 912 Clinical Chemistry System, Roche Diagnostics, Indianapolis, Indiana 46256, USA).

Enrofloxacin $(10 \mathrm{mg} / \mathrm{kg}$ i.v. $)$ was administered in the cephalic vein preoperatively. ${ }^{4}$ Surgical scrub of the biopsy sites was not performed to prevent skin damage or toxic effects with recirculation of con- 


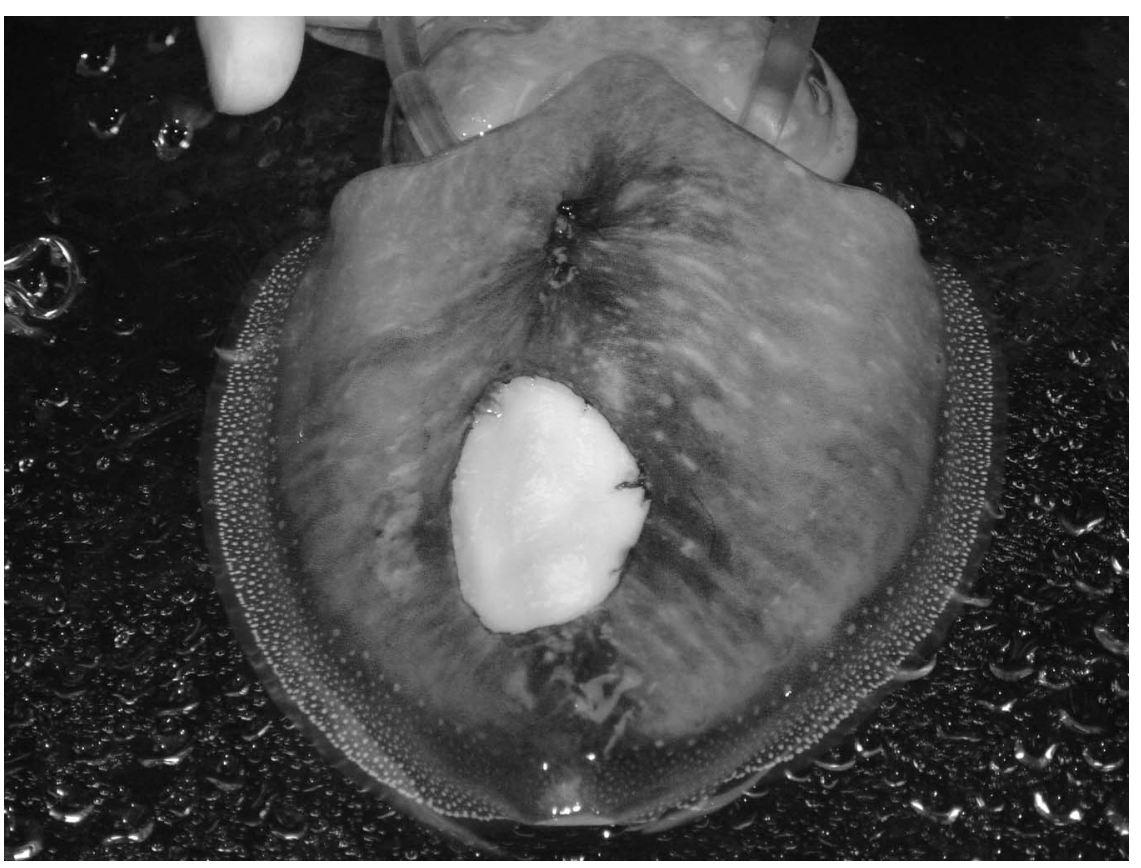

Figure 2. Skin defect following excision of larger caudal granuloma, revealing the underlying white mantle tissue (muscle). The smaller cranial granuloma site has been closed.

taminated anesthesia water. The smaller isolated nodule was removed first, by lifting it by forceps and sharply cutting the stretched skin below the nodule with scissors (Fig. 1). The 5-mm-diameter skin defect was closed with 4-0 polyglyconate on a taper needle $\left(\right.$ Maxon $^{\circledR}, 4-0 \mathrm{CV}-23$ taper, United States Surgical, Norwalk, Connecticut 06850, USA) in a simple continuous pattern. Because the mobility and elasticity of the skin permitted a simple closure with little tension, the same excision procedure was repeated on the larger lesion, resulting in a $3 \times 4$-cm oval skin defect (Fig. 2). The underlying cuttlebone was palpable with instruments, but was completely covered by soft tissue of the mantle. Skin closure with an interrupted cruciate pattern over the larger defect also closed readily, with little tension and no tendency to tear (Fig. 3). A single simple horizontal mattress suture was placed initially, but this caused an eversion of the skin, which was corrected by oversewing with a single simple interrupted suture. The larger nodular lesion adjacent to the ulcer was incised and swabbed for bacterial and fungal culture. All excised tissue was preserved in $10 \%$ neutral buffered formalin for routine histologic processing and staining with hematoxylin and eosin.

Total anesthesia time was $44 \mathrm{~min}$. The cuttlefish was transferred to anesthesia-free seawater for recovery, and respirations were assisted with gentle whole mantle massage. Normal coordinated respirations returned within $2 \mathrm{~min}$. The tentacles (the paired protrusile appendages, versus the eight fixed arms) remained extended and flaccid for $10 \mathrm{~min}$ before they were retracted in response to light pinching. Full recovery was achieved after $20 \mathrm{~min}$ in anesthesia-free water.

Soft-tissue detail was poor on the radiograph, but no radiolucent lytic or irregular areas of the cuttlebone were visualized. Radiographic findings plus observations during lesion excision suggested that the cuttlebone was not affected, which improved the prognosis. Results of hemolymph analysis (Table 1) included hemocytes identified as monocytelike cells with large eosinophilic granules and monomorphic nuclei. Concentrations of some analytes were beyond instrument linearity (sodium, chloride, magnesium), and sample quantity was insufficient to perform dilutions to obtain an accurate result. Sodium, chloride and magnesium hemolymph concentrations are reported in S. officinalis to be $93 \%, 105 \%$ and $98 \%$ of concentrations found in seawater, which are approximately 470, 548, and $54 \mathrm{mmol} / \mathrm{L}$, respectively. ${ }^{16}$ Certain other analytes registered below instrument range (urea nitrogen, creatinine, albumin, gamma-glutamyl transferase, and creatine kinase). Clinical pathology interpretation is hampered by a lack of published reference ranges, and some assays included in the chemistry 


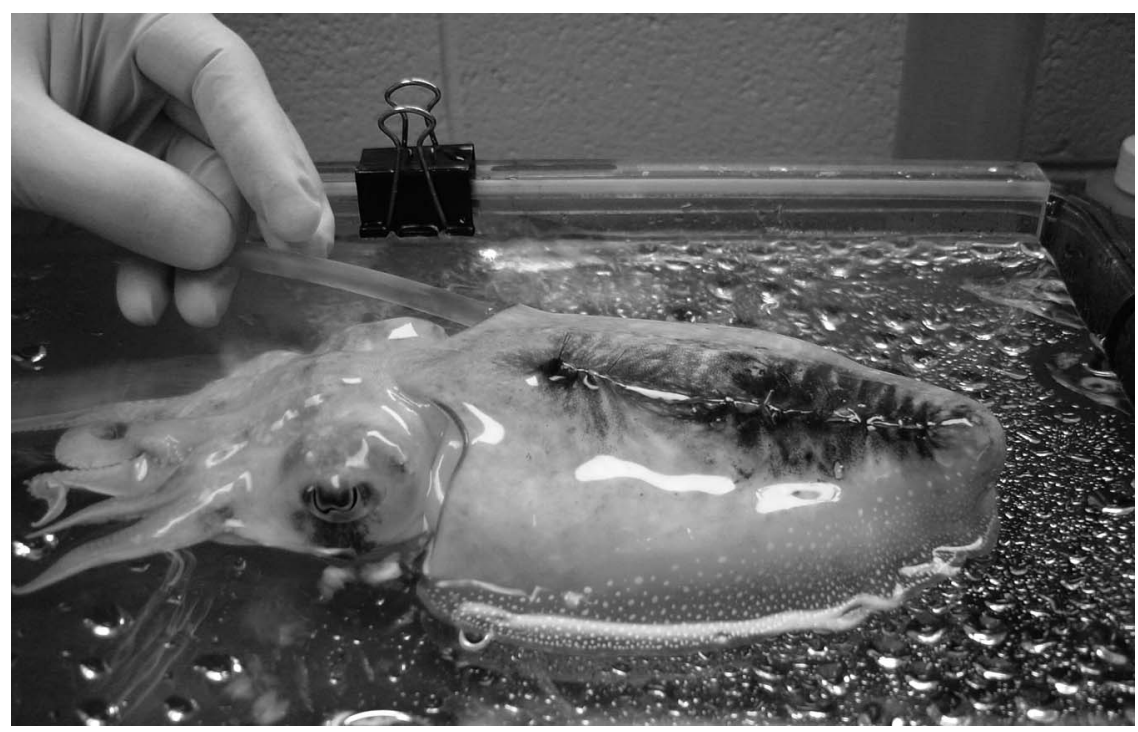

Figure 3. Dorsolateral view of cuttlefish following skin closure. One anesthesia-water delivery tube has been removed for an unobstructed photograph. Notice the color change compared with Fig. 2.

panel have uncertain validity or relevance for an invertebrate.

Bacterial culture yielded heavy growth of Vibrio vulnificus, resistant to amoxicillin, ampicillin, cefazolin, cefoxitin, cephalothin, clindamycin, oxacillin, ticarcillin, and vancomycin, and sensitive to amikacin, cefotaxime, chloramphenicol, ciprofloxacin, erythromycin, gentamicin, imipenem, nitrofurantoin, piperacillin, tetracycline, tobramycin, and trimethoprim-sulfa. Fungal culture yielded heavy growth of Cladosporium sp. Histopathology revealed marked expansion and replacement of the subcutis by multifocal to coalescing granulomas containing a myriad of poorly staining fungal hyphae (Fig. 4). Granulomas were composed of a necrotic eosinophilic center surrounded by a rim of degenerate hemocytes. Adjacent tissues were infil-

Table 1. Hemolymph analysis of an adult female captive cuttlefish with mycotic and bacterial dermatitis.

Hemocyte count $0.50 \times 10^{3} / \mu 1\left(0.5 \times 10^{9} / \mathrm{L}\right)$

Total solids (refractometry) $8.3 \mathrm{~g} / \mathrm{dl}(83 \mathrm{~g} / \mathrm{L})$

Total protein

Glucose

Phosphorus

Calcium

Bilirubin (total)

Alkaline phosphatase

Aspartate aminotransferase

Potassium

Bicarbonate trated with hemocytes admixed with proliferative, swirling spindle cells. A Gomori methenamine silver (GMS) stain revealed abundant long, thin hyphae, 3-5 $\mu \mathrm{m}$ wide with roughly parallel walls, regularly spaced septate, infrequent branching, and occasionally segmental bulbous distensions.

Histologic findings suggested that the fungal infection was the main problem, with local bacterial infection associated with the later ulceration. Based on observations during surgery, it was thought that excision had a reasonable chance of being curative, although the multifocal distribution caused concern about possible systemic distribution. Therefore, itraconazole (Sporonox, Janssen Pharmaceutica Products, Titusville, New Jersey 08560, USA) treatment was initiated at $5 \mathrm{mg} / \mathrm{kg}$ p.o. once daily for 14 days (4-5 granules from a capsule, hidden in food items), with good compliance.

Following surgery, the cuttlefish rapidly returned to normal feeding and activity. Skin color changes were limited to flashing a single eyespot instead of two due to loss of one eyespot location with the excised skin. No other areas of skin swelling developed, and most of the sutures were lost spontaneously, leaving healed incision lines. Two months following surgery the cuttlefish reduced feeding for a few days then was found dead. The whole animal was preserved in $10 \%$ neutral buffered formalin. Histopathology revealed severe multiorgan mycotic infection similar to that described for the skin and mantle biopsy. 

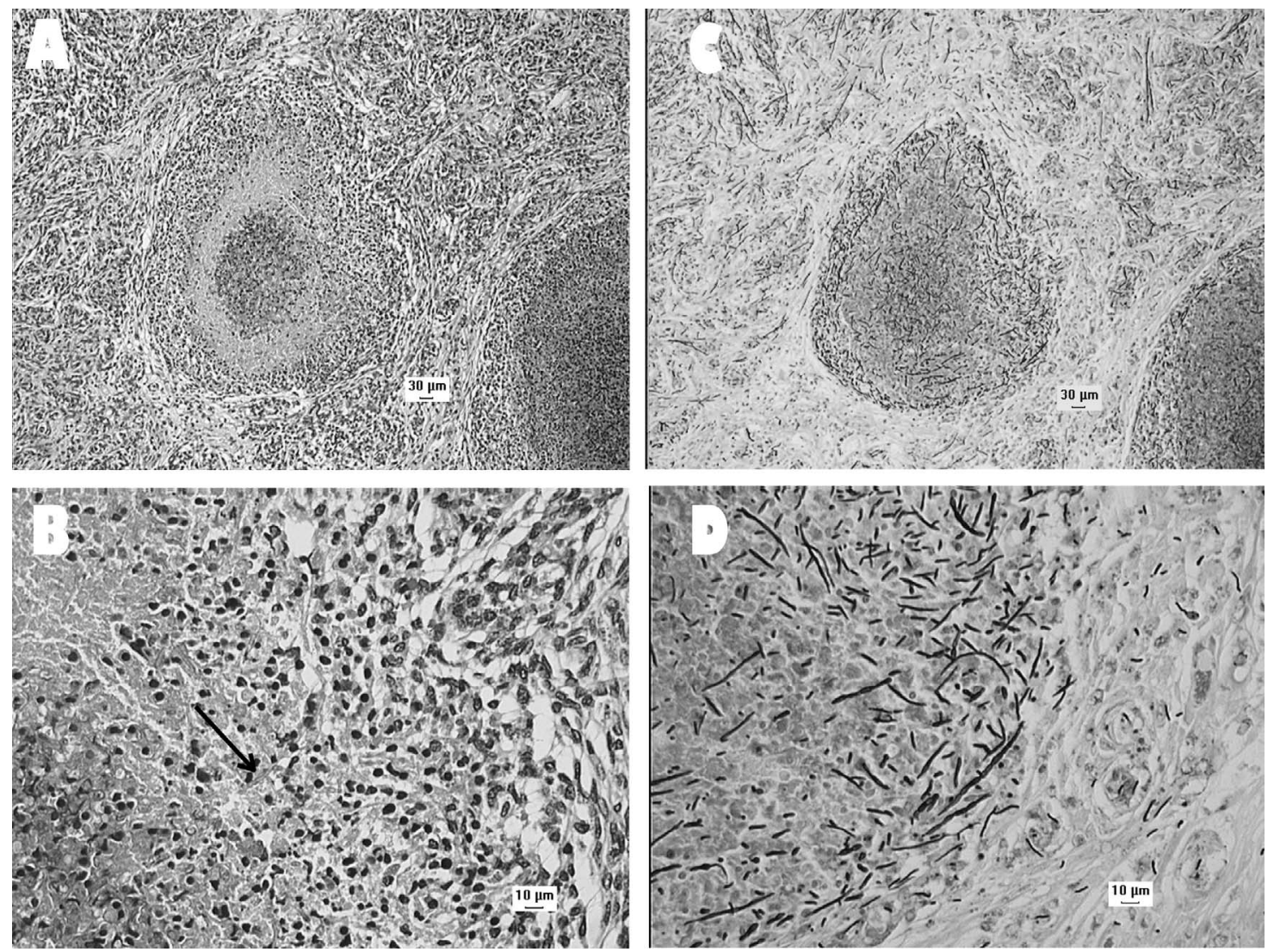

Figure 4. Histopathology of biopsy site, H\&E (a and b) and GMS (c and d) stains, $\times 10$ (a and c) and $\times 40$ (b and d) objectives. Granulomas consist of a necrotic eosinophilic center surrounded by a rim of degenerate hemocytes (a). Adjacent tissue is infiltrated with hemocytes admixed with proliferative spindle cells. Fungal hyphae (arrow in b, and highlighted by GMS stain in $\mathbf{c}$ and $\mathbf{d}$ ) within granulomas are 3-5 $\mu \mathrm{m}$ wide, septate, and branch infrequently.

\section{DISCUSSION}

Cuttlefish skin lesions are typically at the leading edge of the mantle, considered secondary to collision trauma with the tank walls following startle responses during which the animal jets rapidly backward. ${ }^{18}$ As a result, the cuttlebone often fractures or is exposed and can become infected. Minimizing loud noises and sudden movements and providing visual barriers are recommended to reduce occurrence of these injuries. The skin lesions reported here differed in location and progression from those seen as a result of external mantle trauma, appearing instead to originate as a focal eruptive lesion.

Vibrio spp. infections have been reported previously in cephalopods. , $7,10,13,15,17$ These reports include myocarditis in European cuttlefish associated with $V$. alginolyticus, V. parahaemolyticus, or Vibrio sp. myocarditis; and reproductive organ infections in three species of cuttlefish associated with
$V$. alginolyticus, often with concurrent epidermal ulceration; eye, mantle, and other infections in $\mathrm{Eu}-$ ropean cuttlefish associated with Vibrio spp. and other gram-negative bacteria; necrotic exfoliative dermatitis in long-finned squid (Loligo pealei) associated with $V$. anguillarum; and fatal penetrating skin ulcers in young pygmy octopus (Octopus joubini) and reef octopus (O. briareus) in high density culture, associated with $V$. alginolyticus, $V$. damsela (now called Photobacterium damsela), and $V$. parahaemolyticus. In captive brief squids, Lolliguncula brevis, bacterial species composition and concentrations (including Vibrio spp.) were similar between normal and ulcerated mantle, and authors concluded that bacterial infections of ulcerated mantle were secondary to physical trauma or other stressors. ${ }^{2}$ When described histologically, these Vibrio-associated lesions variously include necrosis, edema, hemocyte infiltration, intralesional bacteria, occasional giant cell formation, or necrotizing 
granulomatous-like inflammation. ${ }^{10,13,15}$ Although Vibrio spp. can incite granulomatous-like inflammation in cuttlefish, the abundant fungal hyphae observed within granulomas and subsequent systemic spread of fungal elements in the current case supports the $V$. vulnificus isolated at the time of biopsies having secondary importance in the inflammatory response. The alpha hemolytic Streptococcus $\mathrm{sp}$. isolated prior to presentation was from a surface swab, not reisolated from subsequent deep tissue sampling, and likely a secondary contaminant.

Reports of fungal infections in cephalopods are rare. Fusarium sp. in chambered nautilus and Cladosporium sp. in an unspecified octopus species have been reported but not described in detail. ${ }^{17}$ Cladosporium species are demateacious (pigmented) fungi of the phylum Ascomycota, typically found as saprophytes or plant pathogens, but occasionally occurring as opportunistic skin and systemic infections of humans, the most pathogenic species of which have been transferred to the genus Cladophialophora. 5 Cladosporiosis has been reported in a species of marine tropical fish, the tomato clownfish (Amphiprion frenatus) with deep dermal ulcers, and was thought secondary to immunosuppression from transport or confinement stress. ${ }^{19}$ The inciting cause in the cuttlefish of this report is unknown. No conspecifics housed in the same tank were similarly affected.

The moderately long anesthesia event with surgical excision of dermal fungal granulomas was well tolerated by this cuttlefish, with rapid recovery and incision healing. The skin proved more mobile, elastic and durable than expected, which facilitated sharp surgical excision and skin closure. An unanticipated consequence of the skin excision was the loss of one false eyespot, indicating loss of specific nerve endings, chromatophores or both, which may have limited this cuttlefish's intraspecific signaling repertoire or its ability to startle potential predators.

Necropsy and histopathology findings indicated eventual treatment failure, suggesting that more aggressive excision or more prolonged antifungal treatment would have been warranted. Safety and efficacy of antifungal treatments have not been evaluated in cephalopods. It is unknown whether orally administered itraconazole is adequately absorbed or if long-term administration would be safe in cuttlefish. A formulary of empirically derived treatment protocols for bacterial and protozoal diseases of cephalopods, ${ }^{3}$ and a pharmacokinetic study of enrofloxacin in European cuttlefish ${ }^{4}$ have been published. In the latter study, enrofloxacin clearance was more rapid and volume of distribution was smaller than expected compared with fish and other vertebrates, indicating that extrapolation of vertebrate treatment protocols to invertebrates is uncertain.

Although attempted treatment ultimately failed in this cuttlefish, female European cuttlefish typically live only $12-14$ months, ${ }^{8,18}$ so it was near the end of its expected life span. Senescence may have contributed to recrudescence of the fungal infection. Organism complexity, a suitably large but manageable size, visual appeal, popularity as exhibit animals and utility as laboratory animals would usually favor individual animal veterinary management when indicated for a cuttlefish patient. An inherent drawback of performing more involved medical and surgical procedures on cephalopods, however, is their brief life span. Anesthetic and procedures might still be useful in certain cases, for example, to allow completion of a reproductive cycle. Based on the management of this case, it is concluded that cuttlefish are amenable to extended out-of-water procedures if provided with adequate water circulation over the ctenidia and moisture over the rest of the body, mantle skin is highly mobile and sufficiently strong to hold suture under mild tension, and noneverting suture patterns are suitable for cuttlefish skin closure.

Acknowledgments: We thank Joy Bolynn for photos, Stacey Gore for clinical consultation, Hap Fatzinger for animal care oversight, Donald Meuten and Kevin Woolard for pathology consultation, Michael Dykstra for fungal consultation, Linda Dunn for assistance with figures, and students of the NCSU-CVM Invertebrate Medicine Selective.

\section{LITERATURE CITED}

1. Barnes, R.D. 1980. Invertebrate Zoology, 4th ed. W. B. Saunders Co., Philadelphia, Pennsylvania. Pp. 434466.

2. Ford, L. A., S. K. Alexander, K. M. Cooper, and R. T. Hanlon. 1986. Bacterial populations of normal and ulcerated mantle tissue of the squid, Lolliguncula brevis. J. Invert. Pathol. 48: 13-26.

3. Forsythe, F., R. T. Hanlon, and P. G. Lee. 1990. A formulary for treating cephalopod mollusk diseases. In: Perkins, F. O., and T. C. Cheng (eds.), Pathology in Marine Science. Academic Press, New York, New York. Pp. 5163.

4. Gore, S. R., C. A. Harms, B. Kukanich, J. Forsythe, G. A. Lewbart, and M. G. Papich. 2005. Enrofloxacin pharmacokinetics in the European cuttlefish, Sepia officinalis, after a single i.v. injection and bath administration. J. Vet. Pharmacol. Ther. 28: 433-439.

5. Guarro, J., J. Gene, and A. M. Stchigel. 1999. Developments in fungal taxonomy. Clin. Microbiol. Rev. 12: 454-500. 
6. Hanlon, R. T., and J. W. Forsythe. 1985. Advances in the laboratory culture of octopuses for biomedical research. Lab. Anim. Sci. 35: 33-40.

7. Hanlon, R. T., J. W. Forsythe, and K. M. Cooper. 1984. Fatal penetrating skin ulcers in laboratory-reared octopuses. J. Invert. Pathol. 44: 67-83.

8. Jermann, T. 2001. Captive breeding of Sepia officinalis at the Basel Zoo. Bull. Inst. Oceanogr. 20: 261-265.

9. Lewbart, G. A., and C. A. Harms. 1999. Building a fish anesthesia delivery system. Exotic DVM 1(2): 25-28.

10. Leibovitz, L., T. R. Meyers, R. Elston, and P. Chanley. 1977. Necrolytic exfoliative dermatitis of captive squid (Loligo pealei). J. Invert. Pathol. 30: 369-376.

11. Oestmann, D. J., J. M. Scimeca, J. Forsythe, R. T. Hanlon, and P. Lee. 1997. Special considerations for keeping cephalopods in laboratory facilities. Contemp. Top. 9: 89-93.

12. Reimschuessel, R., and M. K. Stoskopf. 1990. Octopus automutilation syndrome. J. Invert. Pathol. 55: 394400.

13. Reimschuessel, R., M. K. Stoskopf, and R. O. Bennett. 1990. Myocarditis in the common cuttlefish (Sepia officinalis). J. Comp. Pathol. 102: 291-297.

14. Ross, L. G., and B. Ross. 1999. Anaesthetic and
Sedative Techniques for Aquatic Animals, 2nd ed. Blackwell Science, Oxford, United Kingdom. Pp. 46-57.

15. Sangster, C. R., and R. M. Smolowitz. 2003. Description of Vibrio alginolyticus infection in cultured Sepia officinalis, Sepia apama, and Sepia pharaonis. Biol. Bull. 205: 233-234.

16. Schmidt-Nielsen, K. 1990. Animal Physiology: Adaptation and Environment, 4th ed. Cambridge University Press, Cambridge, United Kingdom. Pp. 299-352.

17. Scimeca, J. M. 2006. Cephalopods. In: Lewbart, G. A. (ed.). Invertebrate Medicine. Blackwell, Oxford, United Kingdom. Pp. 79-89.

18. Sherrill, J., L. H. Spelman, C. L. Reidel, and R. J. Montali. 2000. Common cuttlefish (Sepia officinalis) mortality at the National Zoological Park: implications for clinical management. J. Zoo Wildl. Med. 31: 523-531.

19. Silphaduang, J., K. Hatai, S. Wada, and E. Noga. 2000. Cladosporiosis in a tomato clownfish (Amphiprion frenatus). J. Zoo Wildl. Med. 31: 259-261.

20. Stoskopf, M. K., and B. S. Oppenheim. 1996. Anatomic features of Octopus bimaculoides and Octopus digueti. J. Zoo Wildl. Med. 27: 1-18.

Received for publication 5 May 2006 